\title{
A Novel Sand-Dune-Inspired Design for Improved Film Cooling Performance
}

\author{
Wenwu Zhou and Hui Hu ( $\bowtie)$ \\ Department of Aerospace Engineering, Iowa State University \\ 2271 Howe Hall, Ames, IA 50011, USA \\ Corresponding author, Email: huhui@iastate.edu
}

\begin{abstract}
An experimental study was performed to explore a novel film cooling design with a Barchan-DuneShaped Injection Compound (BDSIC) concept for improved film cooling performance over a test plate. Inspired by the unique crescent-shaped Barchan dunes commonly seen in deserts, the BDSIC concept was proposed to enable coolant streams to be discharged tangentially from hollowed Barchan-dune-shaped (BDS) shells and stay attached to the surface of the test plate. During the experiments, while a highresolution Particle Image Velocimetry (PIV) system was used to conduct detailed flow field measurements to quantify the dynamic mixing process between the coolant stream and the mainstream flows over the test plate, a Pressure Sensitive Paint (PSP) technique was used to map the corresponding adiabatic film cooling effectiveness over the surface of interest based on a mass-flux analog to traditional temperature-based cooling effectiveness measurements. The effects of various important design parameters, including the shape of the BDS shells, the blowing ratio of the coolant streams, and the spanwise distance (i.e., the pitch) between the neighboring BDSICs, on the film cooling performance were evaluated in great detail based the quantitative PSP and PIV measurements. It was found that, in comparison with that of conventional film cooling design with coolant stream injected from circular hole (i.e., the baseline case), the film cooling effectiveness over the test plate would be enhanced significantly (i.e., 30\% 500\%) by using the BDSIC design, especially for the test case with elongated BDS shells. As expected, the BDSIC design was found to cause a higher aerodynamic loss at relatively low blowing ratios, due to a greater friction loss associated with its complex shape. However, since the unique BDSIC design could keep the coolant stream to stay attached more firmly on the surface of the test plate (i.e., reducing the aerodynamic loss associated with the penetration of the coolant streams into the mainstream flow), the aerodynamic loss of the BDSIC design was found to become even smaller than that of the conventional film cooling design with circular hole design at relatively high blowing ratios.
\end{abstract}

Keywords: Film cooling; Barchan dune inspired film cooling design; Counter rotating vortex structures; PIV measurements, PSP technique; Mass transfer analog in determining film cooling effectiveness; Aerodynamic loss of film cooling design.

\section{INTRODUCTION}

Thermodynamic analysis reveals that the power output and thermal efficiency of a gas turbine can be further enhanced through a higher turbine inlet temperature, which is now far beyond the maximum endurable temperature of any turbine materials. Therefore, strategies to protect the hot components of gas turbines from melting and burning out are crucial to ensure the sustainable operation of the gas turbines, especially the implementation of film cooling technique. The idea of film cooling is to generate a thin coolant film over the surfaces of hot components to prevent them from direct hot gas exposure, hence, 
increasing their working hours. A real challenge is how to provide essential cooling performance while reducing the consumption of coolant flow in a gas turbine, which requires substantial investigations on film cooling to understand its inherent physics and optimize the designs for better protection of turbine components from harsh environments.

Film cooling, as a state-of-the-art, can be fundamentally equivalent to a jet-in-cross-flow (JICF) problem. As suggested by Fric and Roshko [1], the dominant flow features for a JICF problem include shear-layer vortices formed at the leading edge of the jet, counter-rotating-vortex (CRV) pair in the jet, horseshoe vortices around the jet, and wake vortices downstream of the jet. The performance of a film cooling design is closely related to how firmly a coolant stream can remain attached to the protected surface after injecting into the crossflow. One major problem of film cooling is that, due to the detrimental vortex induction caused by the JICF, the coolant stream tends to take off from the protected surface, leading to a deteriorated cooling performance. In recent years, extensive experimental and numerical studies have been performed to explore novel film cooling designs to alleviate the detrimental vortex induction in JICF, therefore, improving the corresponding film cooling effectiveness. For example, Heidmann et al. [2] and Dhungel et al. [3] introduced an anti-vortex hole concept that could reduce the strength of a CRV pair in the coolant jet. Their measurements showed improved effectiveness of film cooling at certain hole orientation. Zaman and Foss [4] experimentally studied an inclined jet interacting with a triangular tab, and showed that the tab would produce a vorticity cancellation effect that could postpone the jet lifting off from the wall surface. Similar conclusions were also reported by Zaman et al. [5] and Shinn et al. [6], who measured the film cooling effectiveness behind micro-ramp vortex generators. $\mathrm{Na}$ and Shih [7] studied a film cooling configuration with a ramp placed upstream of a coolant hole, and found that a backward-facing ramp can largely enhance the effectiveness of film cooling over the test plate. Zhou and $\mathrm{Hu}$ [8] proposed a Barchan-dune-shaped ramp concept, and found that the detrimental effect of the CRV was partially counteracted by the newly formed anti-CRV pair, leading to improved effectiveness of film cooling behind the dune. Though most of the designs suggested in those previous studies are shown to be effective in improving film cooling performance, some designs with non-streamlined shape could cause increased aerodynamic losses. Therefore, new film cooling designs that not only can provide improved cooling performance, but also with much less aerodynamic loss will be particularly valuable to the gas turbine community.

Barchan dunes are commonly-seen features in deserts. Characterized by a crescentic platform with two gradually-widened horns pointing downstream $[9,10]$, they have streamlined shape in both of the windward (convex face) and leeward side (concave face) along the incoming flow direction. When an air stream flows over it, a pair of symmetric vortices would form in the wake of the dune $[11,12]$. Due to the arched shape of a Barchan dune, the formed vortex pair can generate a continual suction along the axis of horns, creating a "quiet" wake flow behind the dune. As a result, sand particles can settle down on the ground behind the dune, instead of being blown away by the incoming airflow. It should also be noted that, the streamline shaped Barchan dune was found to be able to minimize the aerodynamic losses as airstreams flowing over the dune. Inspired by the unique shape of Barchan dunes, a novel film cooling design with Barchan-dune-shaped injection compound (BDSIC) concept was proposed here to enable coolant streams discharged tangentially from hollowed Barchan-dune-shaped (BDS) shells, and stay more firmly on the surface of the test plate. It should be noted that, the BDSIC design described here can be considered as an upgraded version of BDS ramp concept presented in the earlier work of Zhou \& Hu [8].

In the present study, an experimental investigation was performed to explore a novel film cooling design with BDSIC concept for improved film cooling effectiveness by leveraging the unique flow characteristics behind Barchan dunes. During the experiment, while the coolant stream was injected from a conventional circular hole at an injection angle of $35^{\circ}$, the BDS shell was affixed on the flat plate right above the coolant hole. A Pressure Sensitive Paint (PSP) technique was used to map the corresponding adiabatic film cooling effectiveness on the surface of interest based on a mass-flux analog to traditional temperature-based cooling effectiveness measurements. A high-resolution Particle Image Velocimetry (PIV) system was also used to conduct detailed flow field measurements to quantify the dynamic mixing process between the coolant jet stream and the mainstream flow for the test cases with the BDS shell mounted onto the test plate, in comparison to a baseline case (i.e., with circular hole only). The effects of 
various important design parameters, including the shape of the BDS shells, the blowing ratio of the coolant stream, and the spanwise distance (i.e., the pitch) between the neighboring BDSICs, on the effectiveness of film cooling were evaluated based on quantitative PSP and PIV measurements. Finally, the detailed flow field measurements were correlated with the measured film cooling effectiveness to elucidate the underlying physics pertinent to film cooling in order to explore/optimize design paradigms for better protection of the hot section components in gas turbine.

\section{EXPERIMENTAL SETUP AND TEST MODELS}

\section{Experimental models and test rig.}

The experimental study was conducted in a low-speed, open-circuit wind tunnel located at the Department of Aerospace Engineering of Iowa State University. The wind tunnel has an opticallytransparent test section with a cross section of $200 \mathrm{~mm} \times 125 \mathrm{~mm}$, and is driven by an upstream blower. With honeycombs and screen structures installed ahead of a contraction section, the tunnel can supply uniform low-turbulence incoming airflow into the test section. The turbulence intensity of the mainstream airflow in the test section was found to be $1.5 \%$, as measured by using a hot-wire anemometer.

In the present study, all the components of the test model, including a test plate and BDS shells, were made of a hard plastic material and manufactured by using a rapid prototyping machine that built the model components layer-by-layer with a resolution of about 25 microns. Figure 1 (a) shows the BDSIC film cooling concept, where the BDS shell was affixed on the surface of the test plate by using a layer of $80 \mu \mathrm{m}$-thick double-sided glue tape. For the baseline case (i.e., with conventional circular hole only), the BDS shell was removed from the test plate. The diameter $(D)$ and injection angle of the circular hole on the test plate were $12 \mathrm{~mm}$ and $35^{\circ}$, respectively. While the inlet and outlet of coolant hole were sharpedged, the internal path of the coolant hole (i. e., with $2.5 D$ entry length) was carefully polished with a 2000 grit sand paper. As shown clearly in Fig. 1 (a), the axial centerline of the circular part would intersect the upper surface of test model at $144 \mathrm{~mm}$ downstream from the leading edge of the plate model. As a result, before interacting with the coolant stream, the boundary layer of mainstream flow would develop over a length of $12 \mathrm{D}$. During the experiment, while the test plate was flushing mounted onto the bottom wall of test section, a plenum chamber $(115 \mathrm{~mm} \times 115 \mathrm{~mm} \times 90 \mathrm{~mm})$ was sited underneath the test plate and sealed by a thin latex rubber gasket.

Figure 1 (b) shows the geometric dimensions of different BDS shells, i.e., shortened, standard, and elongated BDS shells, used in the present study to implement the proposed BDSIC film cooling concept. It should be noted that, due to the environmental diversity, the size and shape of the Barchan dunes existing in nature were found to vary in a wide range. In the present study, the exterior geometry of the standard BDS shell was generated from a stereoscopic topographic map of an idealized Barchan dune reported in previous studies $[9,11,13,14]$. The internal shape geometry of BDS shell has a similar shape as the exterior geometry of the idealized sand dune. The shortened BDS shell was obtained by reducing the length of the BD horns of the standard BDS shell (i.e., $L_{a}=1.8 D$ ) to $L_{a}=1.3 D$, but with all the other dimensions being the same as the standard BDS shell case. A similar method was also applied for the generation of the elongated BDS shell with the front part (i.e., $L_{0}$ ) of the BDS shell being extended to be $2.0 D$ to ensure a full coverage of the coolant injection hole in the BDSIC configuration. In the present study, the coolant streams exhausted from the BDS shells were designed to inject tangentially into the mainstream flow, which can consequently minimize the mixing loss. It should also be noted that, the BDS shells were designed to have the same exit area as that of the conventional circular hole.

During the experiment, while the airflow from the wind tunnel was used to simulate the hot gas flow in a typical turbine stage, an oxygen-free gas (i.e., $\mathrm{CO}_{2}$ with a density ratio of $D R=1.5$ for the present study) was supplied to simulate the "coolant" stream for the PSP measurements. The coolant gas, supplied by a pressurized $\mathrm{CO}_{2}$ gas cylinder (99.99\% purity), passed through a long pipeline and a constant temperature thermal bath before finally entering into the plenum chamber underneath the test 
plate. The blowing ratios (i.e., mass flux ratios, $M=\rho_{c} U_{c} / \rho_{\infty} U_{\infty}$ ) of the test cases were monitored by a flow meter (Omega, FMA-1600A), varied from 0.60 to 1.50. The corresponding momentum ratios $\left(I=\rho_{c} U_{c}^{2} / \rho_{\infty} U_{\infty}^{2}=M^{2} / D R\right.$ ) were found to range from 0.24 to 1.50 , and the velocity ratios $\left(V_{r}=U_{c} / U_{\infty}\right)$ being changed from 0.4 to 1.0 .

In the present study, the mainstream flow velocity at the inlet of the test section was fixed at a constant speed of $U_{\infty}=25 \mathrm{~m} / \mathrm{s}$. A stripe of 2.0 inches wide and 32 grid sand paper was employed at the leading edge of the test section to trip the incoming boundary layer to ensure a fully-developed turbulent boundary layer airflow over the flat surface. The boundary layer profile was measured immediately upstream of the coolant holes (i.e., without BDS shells mounted onto the test plate), which was found to have $\delta_{99} \approx 0.72 D$, corresponding momentum thickness $\theta \approx 0.08 D$ (i.e., the corresponding Reynolds number of $R e_{\theta} \approx 1.5 \mathrm{~K}$ ), and the shape factor, $H \approx 1.34$.

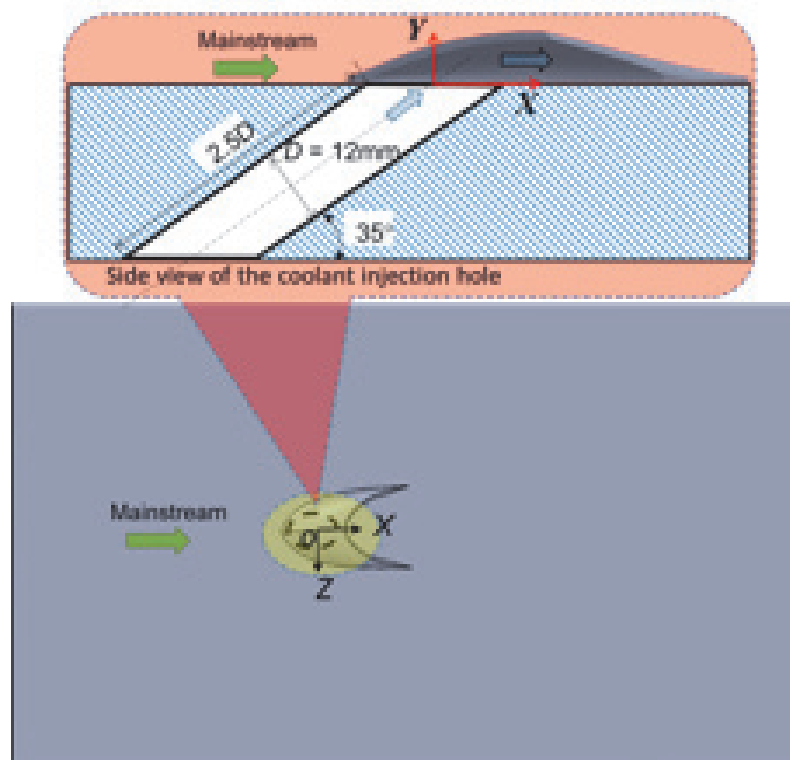

(a)
BDSIC film cooling design

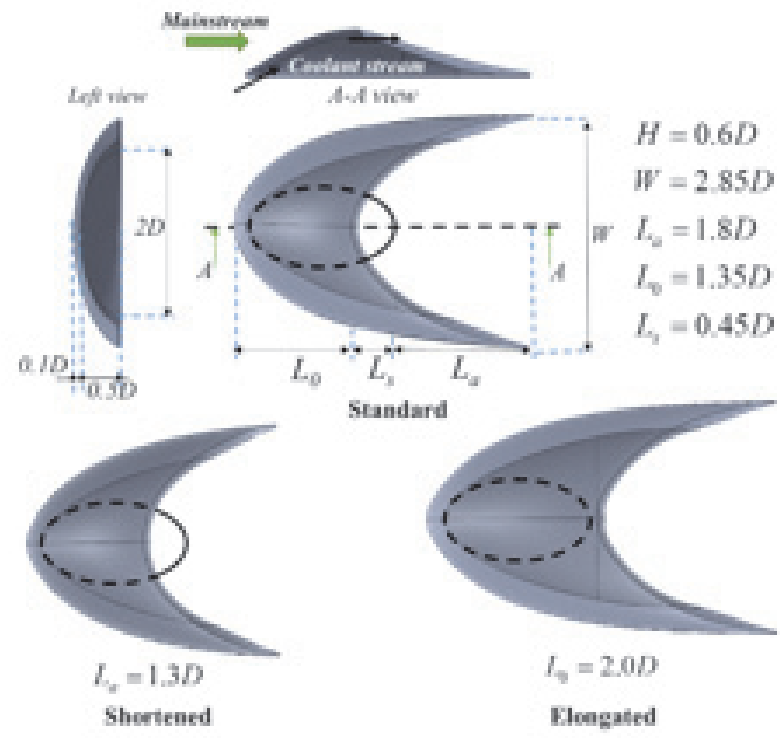

(b)

Detailed dimensions of the BDS shells

Figure 1. The schematic of BDSIC film cooling concept, where $D$ is the diameter of coolant injection hole.

\section{Adiabatic film cooling effectiveness measurement by using PSP technique.}

Traditionally, adiabatic film cooling effectiveness, $\eta$, is expressed as,

$$
\eta=\frac{T_{\infty}-T_{a w}}{T_{\infty}-T_{c}}
$$

where $T_{\infty}$ is the temperature of the mainstream flow, $T_{a w}$ is the adiabatic wall temperature under inspection, and $T_{c}$ is the temperature of the coolant stream at the hole exit. Obviously, the primary challenge associated with the temperature-based methods lays in measuring the true adiabatic wall temperature, in spite of the physical reality of heat conduction through the test model.

When the Lewis number $\left(L e=\alpha / D_{s}\right.$, where $\alpha$ is thermal diffusion, and $D_{s}$ is concentration diffusion coefficient) for the coolant flow is approximately 1.0, the thermal boundary layer and concentration 
boundary layer thickness would be of the same order, implying that the differential equations involving heat and mass transfer would be analogous [15-17]. Based on a mass transfer analogy, Pressure Sensitive Paint (PSP) technique has widely used in recent years to measure film cooling effectiveness on the surface of interest. Since PSP measurements can usually be conducted under isothermal conditions, the concerns and implications due to the effects of heat conduction through the test model can be eliminated easily.

In PSP experiments, the surface of interest would be coated with a layer of oxygen-sensitive paint, which consists of luminophore molecules. When excited by certain UV light, the luminophore molecules would emit photoluminescence. However, the excited molecules may return to the ground state via a reduced or radiationless emission in the presence of diatomic oxygen molecules. This process is usually called oxygen quenching [18], and the intensity of the photoluminescence is inversely proportional to the concentration of the local oxygen. Consequently, the concentration of oxygen over the interested surface can be calculated based on the recorded light intensity through the use of a calibration curve [19]. Applying the concentrations of oxygen rather than temperatures into Eq. (1), the film cooling effectiveness can be expressed as Eq. (2), as suggested by Charbonnier et al [20], where $M W$ is the molecular weight ratio of the coolant gas to the mainstream gas.

$$
\eta=1-\frac{1}{\left[\left(\left(p_{o_{2}}\right)_{\text {air }} /\left(p_{o_{2}}\right)_{\text {mix }}\right)_{\text {wall }}-1\right] M W+1}
$$

The pressures in Eq. (2) can be determined by using the recorded intensity of emitted photoluminescence light, which is inversely proportional to the partial pressure of oxygen. The mathematic function between the normalized intensity and partial pressure can be obtained through a dedicated PSP calibration procedure. Further technical details about PSP technique and calibration procedure for film cooling effectiveness measurements can be found in the references of $[17,19,21]$.

It should be noted that, Johnson et al. [19] and Zhou \& Hu [8] performed comparative studies of the measured film cooling effectiveness by using PSP technique against those derived from temperaturebased measurements under same or comparable conditions. It was found that the measured effectiveness of film cooling with PSP technique agrees well with those derived from temperature-based measurements. Applying the similar experimental setup as those used in Johnson et al. [19] and Zhou \& Hu [8], the PSP technique was utilized in the present study to map the film cooling effectiveness distribution over the surface of interest at various test conditions. Figure 2 shows the schematic of the experimental setup used for the PSP measurements. A constant UV LED light (LM2X-DM, ISSI) with wavelength of $390 \mathrm{~nm}$ was used as the excitation source for the PSP measurements. A 14-bit $(2048$ pixel $\times 2048$ pixel $)$ chargecoupled device (CCD) camera (PCO2000, Cooke Corp.) with a $610 \mathrm{~nm}$ long-pass filter was used to records the intensity of the photoluminescence light emitted by excited PSP molecules. The PSP paint used in the study was Uni-FIB provided by ISSI, which has a low sensitivity to temperature variation $(\sim 0.5 \% /$ $\left.{ }^{\circ} \mathrm{C}\right)$. The experimental study was conducted at an isothermal condition with the environment temperature being held at constant temperature of $22 \pm 0.5^{\circ} \mathrm{C}$.

In the present study, a spatially-averaging procedure with interrogation windows of 9 pixels $\times 9$ pixels and a $50 \%$ overlap rate was performed for t PSP image processing in order to minimize the random noises on the PSP measurements. The acquired PSP images have a magnification of $0.085 \mathrm{~mm} / \mathrm{pix}$, which results in a spatial resolution of $0.34 \mathrm{~mm}$ or $0.03 D$ for the PSP measurement results. It should be noted that the uncertainty of measured film cooling effectiveness by using PSP technique is closely related to PSP calibration process, quality of the acquired raw images, and the flow conditions of the mainstream and coolant streams. Following up the work of Johnson \& $\mathrm{Hu}$ [22], the absolute measurement uncertainty of the present study was estimated to be within $\Delta \eta=0.02$ for the measured centerline film cooling effectiveness. The relative measurement uncertainty is estimated to be within $3 \%$ for $\eta=0.6$ and $5 \%$ for $\eta=0.4$. As for the laterally-averaged film cooling effectiveness, the absolute uncertainty would be within 
$\Delta \eta=0.03$, and the corresponding relative uncertainty is within $5 \%$ for $\eta=0.6$ and $7 \%$ for $\eta=0.4$. These uncertainty values were estimated based on a Taylor Series Method [23,24].

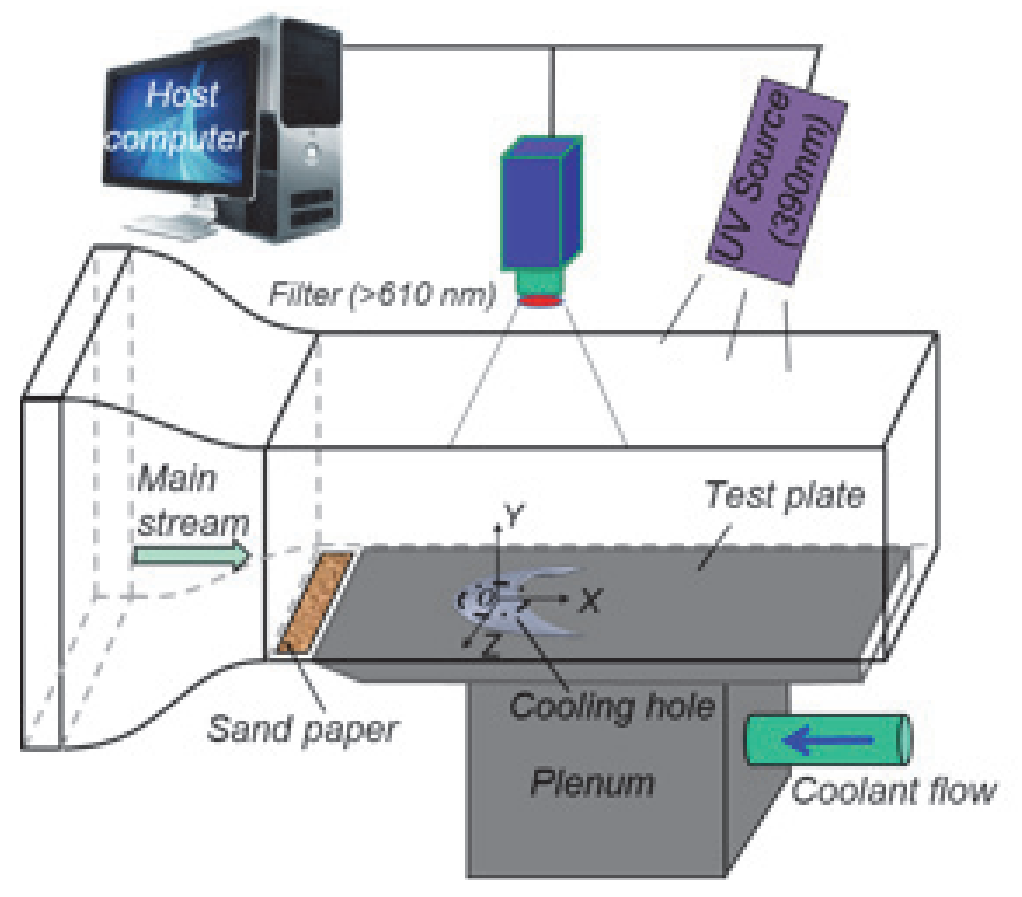

Figure 2. Experimental setup used for the PSP measurements.

\subsection{Flow field measurements by using the PIV and stereoscopic PIV (SPIV) technique.}

A high-resolution PIV system was used to conduct detailed flow field measurements to quantify the dynamic mixing process between the coolant and mainstream flows over the test plate. Figure 3 shows the schematic of the experimental setup for the PIV measurement. During the experiment, the mainstream airflow and the cooling jets (i.e., $\mathrm{CO}_{2}$ for the present study) were seeded with $\sim 1 \mu \mathrm{m}$ oil droplets generated by droplet generators. Illumination was provided by a double-pulsed Nd:YAG laser (NewWave Gemini 200), adjusted on the second harmonic and emitting two pulses of $200 \mathrm{~mJ}$ with the wavelength of $532 \mathrm{~nm}$ at a repetition rate of $5 \mathrm{~Hz}$. Using a set of high-energy mirrors and optical lenses, the laser beam was shaped into a thin light sheet with thickness of about $1.0 \mathrm{~mm}$ in the measurement interest. The illuminating laser sheet was firstly aligned along the mainstream flow direction, bisecting the coolant hole in the middle of the test plate, to conduct 2D PIV measurements in the $X-Y$ plane. Then, the laser sheet was rotated $90^{\circ}$ to perform stereoscopic PIV (SPIV) measurements at different downstream locations behind coolant exit to reveal the flow structures in the cross planes (i.e., $Y-Z$ planes).

In the present study, two 14-bit high-resolution CCD cameras (PCO2000, Cooke Corp.) were used for the SPIV image acquisitions. The two cameras were arranged with an angular displacement configuration of about 45 degrees to get a largely overlapped view. With the installation of tilt-axis mounts, the laser illumination plane, the lenses, and the camera bodies were adjusted to satisfy the Scheimpflug condition. The CCD cameras and double-pulsed Nd:YAG laser were both connected to a Digital Delay Generator (Berkeley Nucleonics, Model 565) to control the timing of the lasers and image acquisitions. A general in-situ calibration procedure was conducted to obtain the mapping functions between the image planes and object planes for the SPIV measurements. A target plate $\left(\sim 150 \times 100 \mathrm{~mm}^{2}\right)$ with $0.5 \mathrm{~mm}$ diameter dots spaced at intervals of $1.0 \mathrm{~mm}$ was used for the in-situ calibration. The mapping function used in the present study was a multi-dimensional polynomial function, which is fourth order for the directions parallel to the laser illumination plane (i.e., $Y$ and $Z$ directions), and second order for the direction normal 
to the laser sheet plane (i.e., $X$ direction). The coefficients of the multi-dimensional polynomial were determined from the calibration images by using a least-square method as used by $\mathrm{Hu}$ et al. [25].

For the 2D PIV measurements in the streamwise plane, instantaneous flow velocity vectors were obtained by using a frame-to-frame cross-correlation technique to process the acquired PIV images with an interrogation window size of 32 pixels $\times 32$ pixels. An effective overlap of $50 \%$ of the interrogation windows was employed in PIV image processing, which resulted in a spatial resolution of $0.7 \mathrm{~mm}$ (i.e., $~$ $0.06 D$ ) for the PIV measurement results. Similar process methodology was also used for SPIV image processing. The instantaneous $2 \mathrm{D}$ velocity vectors were then used to reconstruct all three components of the flow velocity vectors in the laser illuminating plane by using the mapping functions obtained through the calibration procedure. After the instantaneous flow velocity vectors $\left(u_{i}, v_{i},{ }^{w_{i}}\right)$ were determined, the distributions of the ensemble-averaged flow quantities such as mean velocity $(U, V, W)$, normalized turbulence kinetic energy $\left(0.5\left(\overline{u^{2}}+\overline{v^{2}}+\overline{w^{2}}\right) / U_{\infty}^{2}\right)$, spanwise vorticity $\left(\omega_{z}=\frac{\partial v_{i}}{\partial x}-\frac{\partial u_{i}}{\partial y}\right)$ for the 2D PIV measurements, and streamwise vorticity $\left(\omega_{x}=\frac{\partial w_{i}}{\partial y}-\frac{\partial v_{i}}{\partial z}\right)$ for the SPIV measurements were obtained from a sequence of 1,000 frames of instantaneous PIV measurement results. The uncertainty level for the PIV measurements is estimated to be within 3\% for the instantaneous velocity vectors, while the uncertainties for the measured ensemble-averaged flow quantities such as vorticity distributions are about $10 \%$.

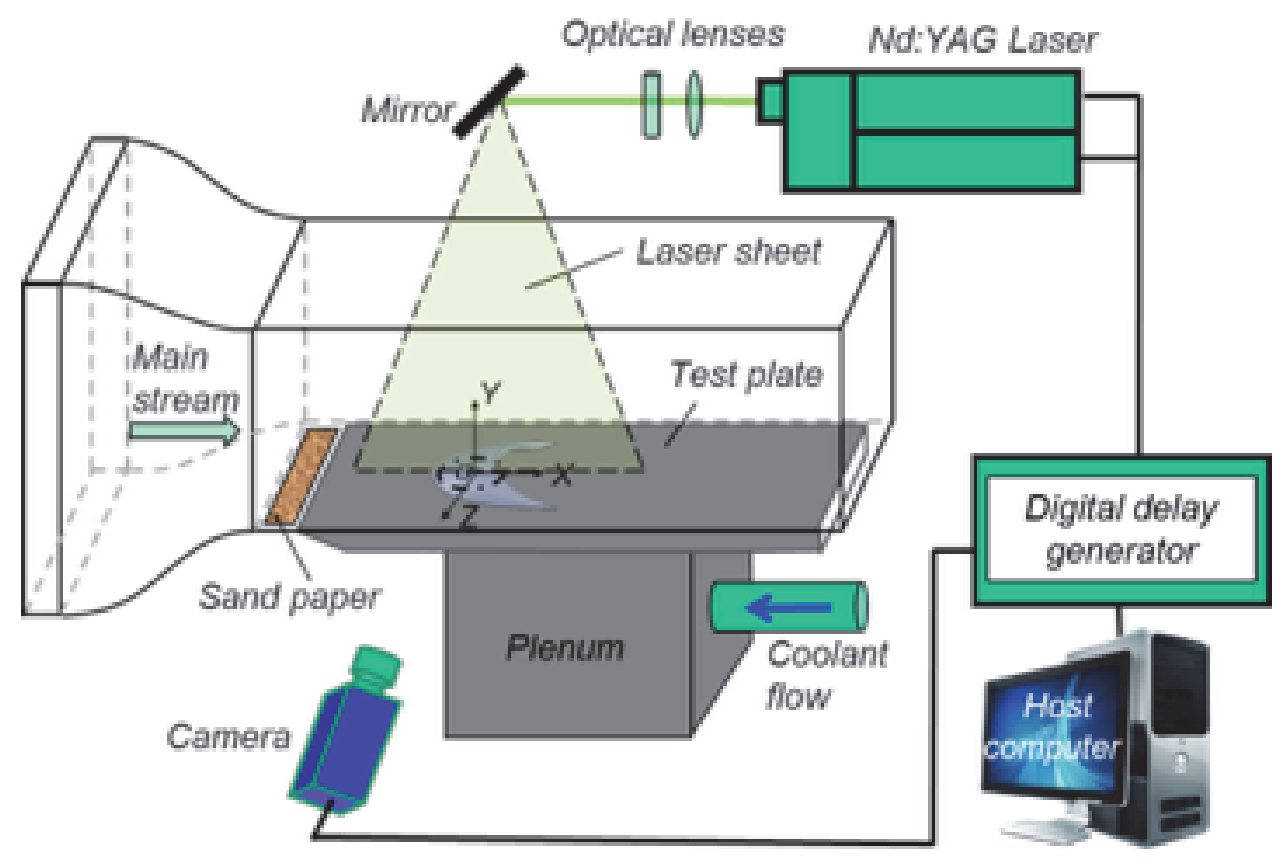

Figure 3. Experimental test rig for PIV measurements.

\section{Measurement Results and Discussions}

\subsection{Effects of the BDS shells on the film cooling effectiveness}

Figure 4 shows the film cooling effectiveness distributions over the test plate for the proposed BDSIC film cooling designs (i.e., with different BDS shells, including shortened, standard, and elongated shells) at various blowing ratios, in comparison with those of the baseline cases (i.e., the conventional circular hole case without BDS shell). As shown clearly in Fig. 4, the film cooling effectiveness for the BDSIC cases were found to be much higher than those of the baseline cases at the blowing ratios of $M=0.6,0.9$ 
and 1.2. At the relatively low blowing ratio (i.e., $M=0.6$ ), though the baseline case was found to exhibit a decent film coverage, the appearance of BDS shells was found to greatly improve the coverage of coolant over the surface of interest. As the blowing ratios increased to relatively high values (i.e., $M \geq$ 0.9 ), the regions with high cooling effectiveness for the baseline cases were found to become shorter and narrower, which indicates the high momentum coolant streams having token off from the surface of the test plate. On the contrary, the film cooling effectiveness for the BDSIC cases were found to increase monotonically as the blowing ratio increase. It should be noted that, the film cooling performance for the test case with elongated shell was found to be the best among all the tested BDSIC designs. In general, the measured film cooling effectiveness results suggest that the existence of BDS shells are very beneficial for improving the film cooling performance over the test plate, especially for the BDSIC design with elongated shell.

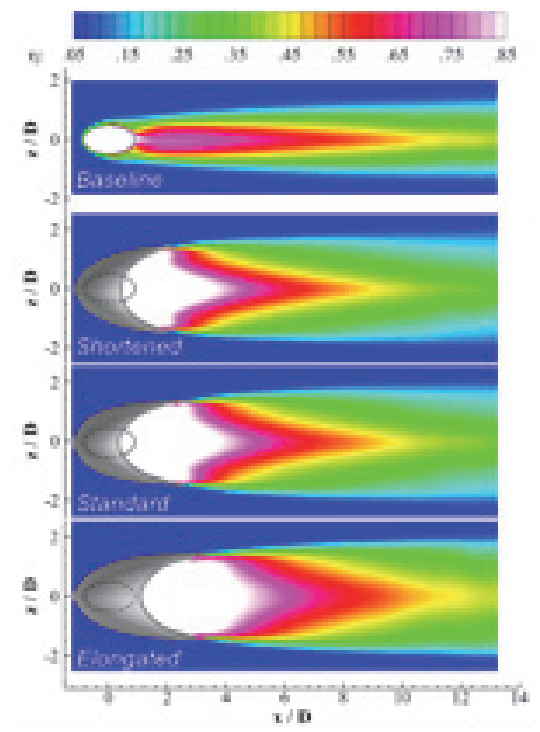

(a). $M=0.6$

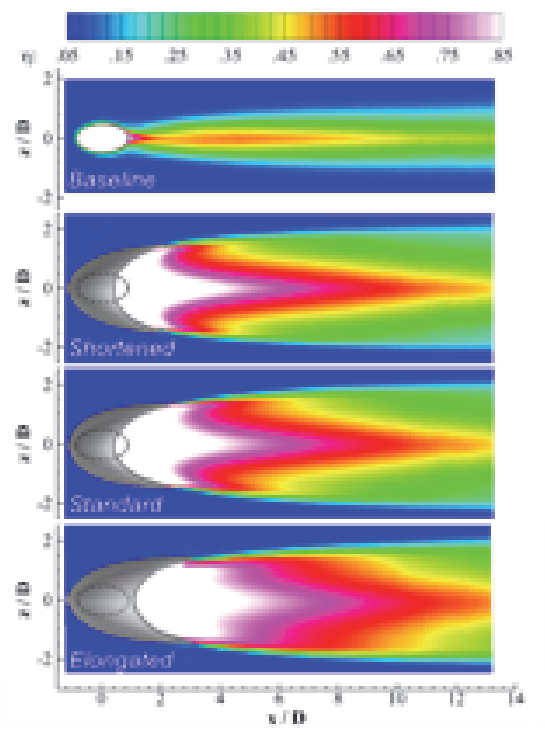

(b). $M=0.9$

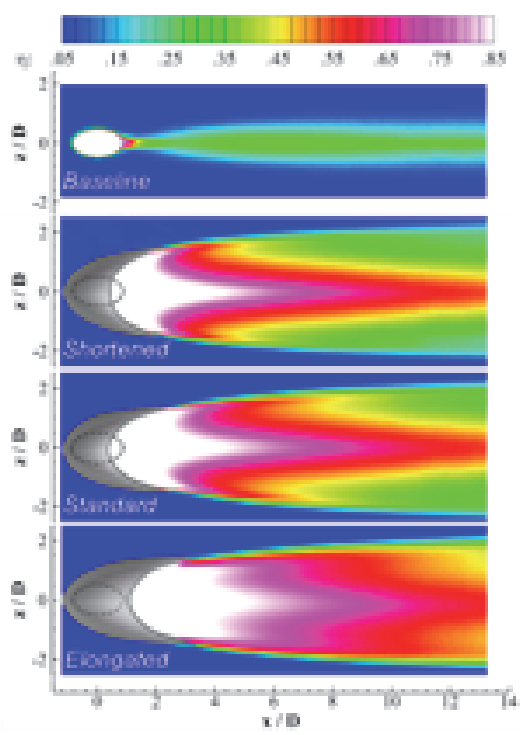

(c). $M=1.2$

Figure 4. Measured film cooling effectiveness distributions for the baseline and the BDSIC designs at various blowing ratios.

Based on the measured cooling effectiveness distributions shown in Fig. 4, the centerline as well as the laterally-averaged cooling effectiveness profiles were extracted and replotted in Fig. 5, which were used to reveal the effects of the BDS shells on the film cooling performance more clearly and quantitatively. The centerline effectiveness profiles (i.e., $\left\langle\eta_{z=0}\right\rangle$ ) refer to the measured cooling effectiveness values along the centerline of the coolant injection hole, while the laterally-averaged effectiveness profiles (i.e., $\left\langle\eta_{|z| \leq 1.5 D}\right\rangle$ ) are determined by averaging the measured cooling effectiveness along the spanwise direction over one full period of the hole spacing (i.e., in the region of $-1.5 \leq Z / D \leq$ 1.5). It is worth noting that, while similar results were also found at other blowing ratios, only the measured results with the blowing ratios of $M=0.6$ and 1.2 are shown here for conciseness. As shown clearly in Fig. 5, the measured cooling effectiveness (i.e., including both the centerline and laterallyaveraged profiles) for the test cases with BDSICs were found to be significantly higher than those of the baseline case, which is consistent with the characteristics revealed in the spatial contour distributions shown in Fig. 4. This is largely due to the fact that, as jet momentum increases, the coolant stream for the baseline case tends to take off from the surface of the test plate and penetrate into the mainstream flow. However, the unique BDSIC designs would suppress the take-off of the coolant flow and make it stay firmly over the surface of the test plate. 

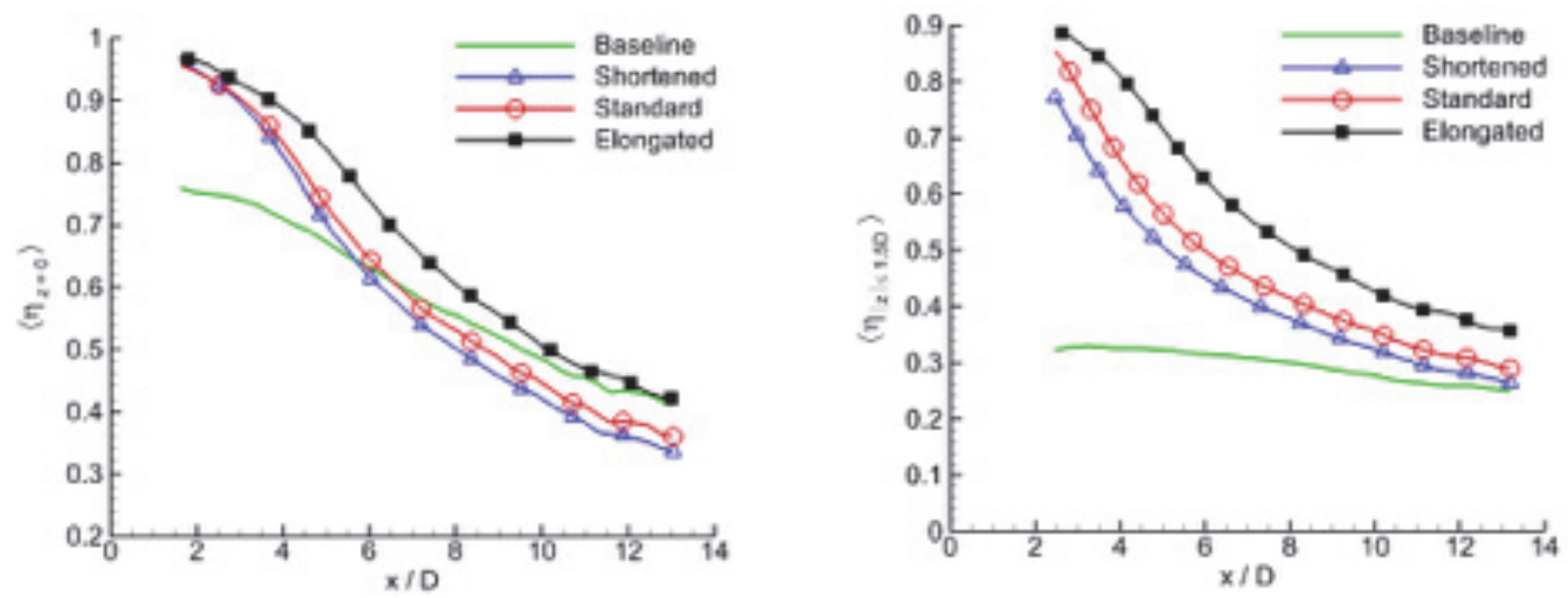

(a). Measured cooling effectiveness profiles at $M=0.6$
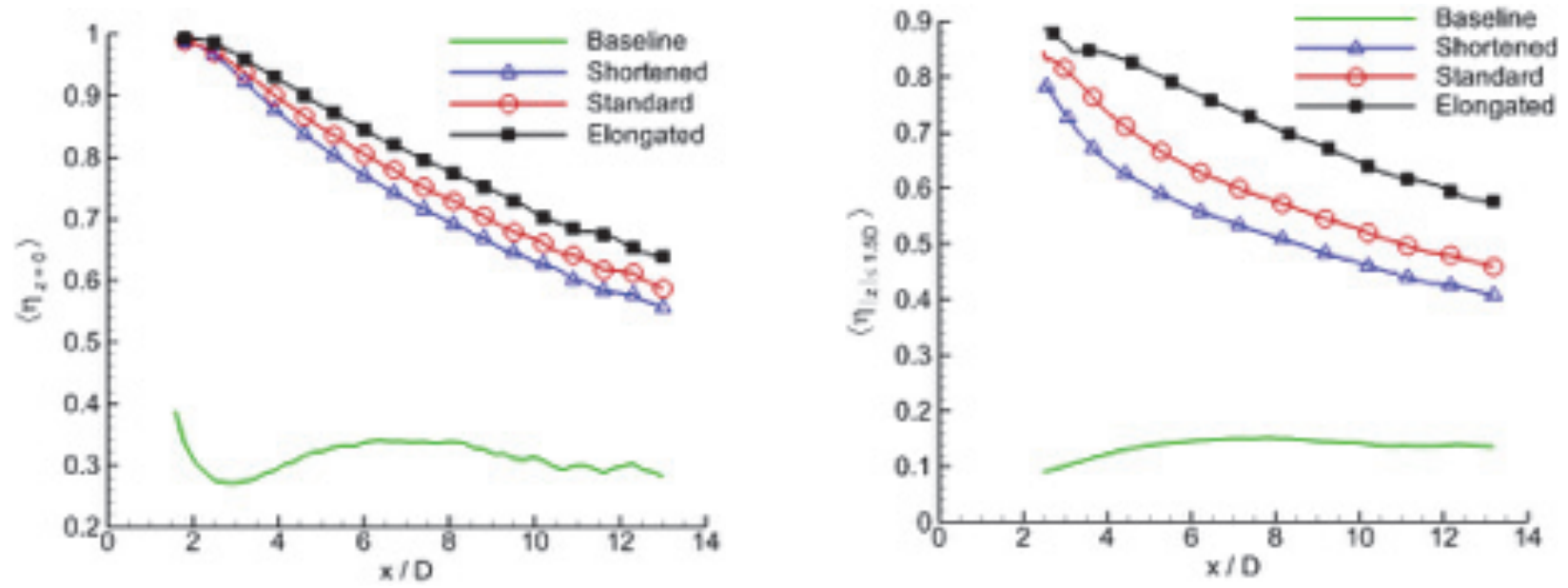

(b). Measured cooling effectiveness profiles at $M=1.2$

Figure 5. Comparison of the cooling effectiveness profiles between the BDSIC designs and baseline case at the blowing ratios of $M=0.6$ and $M=1.2$.

Based on the measured results given in Fig. 5, it can also be seen clearly that the effectiveness of film cooling for the test case with the elongated BDS shell was found to be the highest among all the tested BDSICs, followed closely by the case with standard BDS shell, and finally the shortened BDS shell case. For the test cases with the BDSIC, the coolant stream bled from the circular tube would impinge onto the inner front part of dune at first, and then discharge into the cross-flow. During this process, the length of the front part of BDS shell would be a critical parameter that will affect the behavior of the coolant jet stream. The BDSIC design with longer front length could completely avoid the separation of the coolant jet stream from the test surface, otherwise a portion of the coolant stream may take off from the test plate and penetrate into the mainstream flow, resulting in reduced cooling performance. In the present study, the design with the elongated BDS shell has the longest front part of the shell, which consequently leads to the highest effectiveness behind the coolant injection hole among the three tested cases.

In general, the existence of the elongated BDS shell for the BDSIC design would greatly improve the effectiveness of the film cooling over the surface of interest. More specifically, in comparison to the baseline case, an augmentation of $30 \% \sim 500 \%$ in laterally-averaged cooling effectiveness was observed 
for the elongated BDS shell case at the blowing ratios of $M=0.6 \sim 1.2$. Since the elongated BDSIC design was found to exhibit the best cooling performance among all the test cases, this configuration was chosen for further investigation to examine the underlying physics pertinent to the improved film cooling performance. From now on, if without any specification, the measurement results given in the present study would refer to the BDSIC design with the elongated BDS shell.

\subsection{2-D PIV measurement results for the BDSIC and baseline cases.}

As described above, a high-resolution 2D PIV system was used in the present study to conduct detailed flow field measurements to quantify the dynamic mixing between the mainstream and coolant flows, where the laser sheet was aligned along the middle plane of coolant hole (i.e., $X$ - $Y$ plane as shown in Fig.3). Figure 6 shows the PIV measurement results for the baseline case (left column) and the BDSIC design with the elongated BDS shell at the blowing ratio of $M=0.9$, where the $\mathrm{CO}_{2}$ gas (i.e., $D R=1.5$ ) was used as the coolant in the present study. As revealed clearly in Fig. 6 (a), the coolant jet stream was found to mix intensively with the mainstream flow over the test plate, which would dilute the potential of coolant stream to protect the surfaces of the turbine blades from hot gas stream in gas turbine. Due to the Kelvin-Helmholtz instabilities [26,27] within the shear layer between the coolant and mainstream flows, packets of unsteady vortex structures were found to be generated along the interface between the two streams for both circular hole and BDSIC cases.
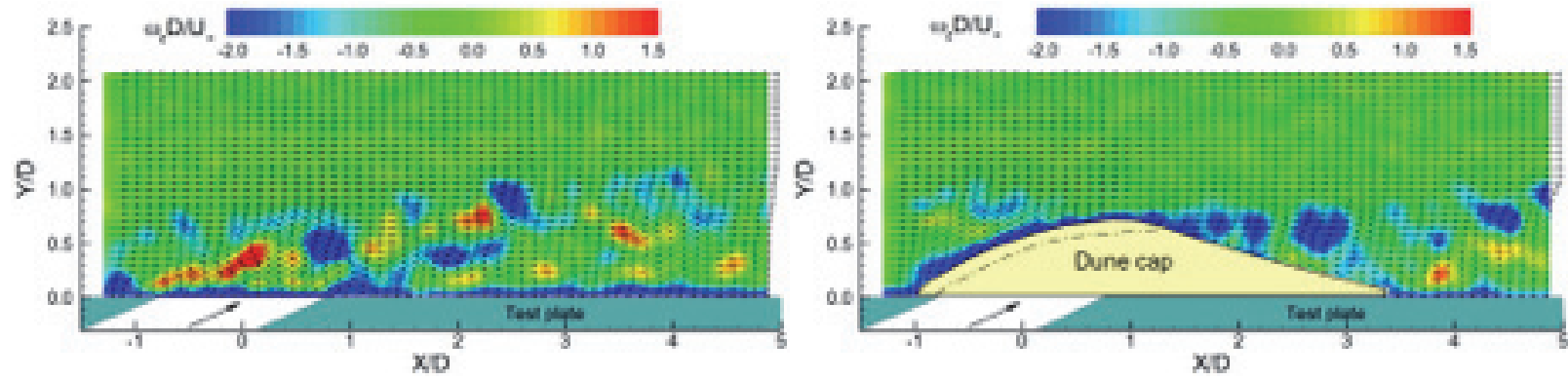

(a) Instantaneous vorticity distributions
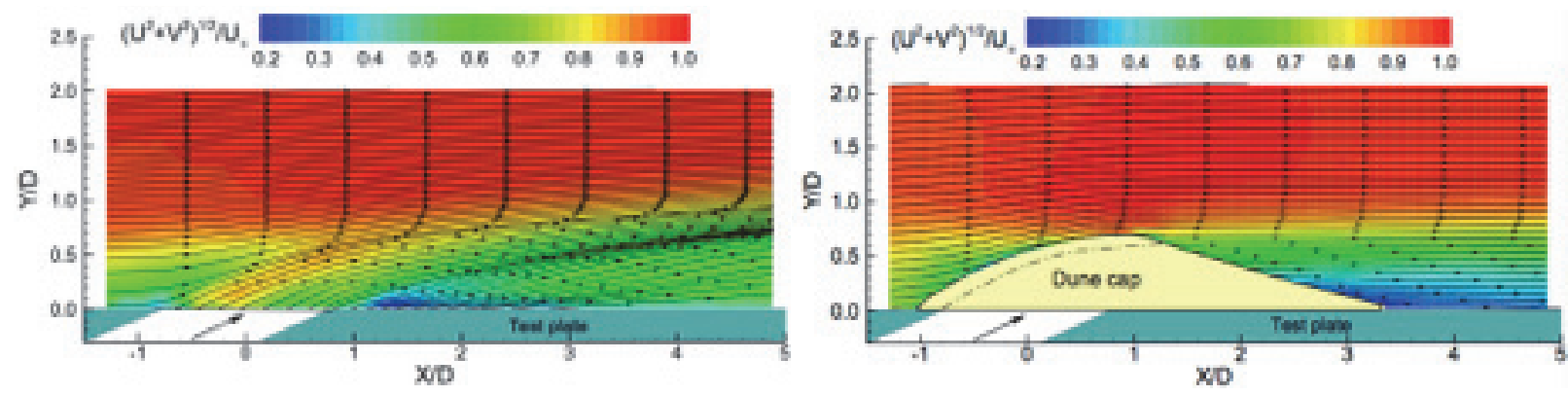

(b) ensemble-averaged velocity field

Figure 6. PIV measurement results for the test cases of baseline (left) and BDSIC design with elongated shell (right) at the blowing ratio of $M=0.9$.

As shown clearly in Fig. 6 (b), while the coolant stream exhausted from the conventional circular hole for the baseline case was found to separate from the test plate, the coolant stream injected from the BDSIC with the elongated BDS shell was found to discharge tangentially into the mainstream flow and stay on the surface of test plate nicely. Due to the existence of the BDS shell, the high momentum jet stream would stay firmly on the surface of the test plate as it exhausted from coolant injection hole. This observation also indicates that the cooling effectiveness for the BDSIC case would be significantly higher than that of the baseline case, which is consistent with the measured film cooling effectiveness results shown in Fig. 5. 


\subsection{Stereoscopic PIV measurement results for the BDSIC and baseline cases.}

As discussed above, the most prominent features in a JICF would include jet shear layer vortices formed at the leading edge of jet, horseshoe vortices around the jet, CRV pair within the jet, and wake vortices downstream of jet flow [1]. As shown clearly in the PIV measurement results given in Fig. 6, the coolant jet stream for the baseline case was found to take off from the test plate and penetrate into the mainstream flow due to the induction from the strong CRVs, which leads to deteriorated film cooling performance over the surface of plate. As suggested by Heidmann et al. [2], Dhungel et al. [3], Zaman et al. [5], Shinn et al. [6], and Zhou and $\mathrm{Hu}$ [29], one effective method to improve the film cooling performance is to reduce the detrimental vortex induction by introducing a pair of anti-CRV to weaken its strength, therefore postponing the separation of the coolant jet from the surface of interest.

To elucidate the underlying physics pertinent to film cooling, a stereoscopic PIV (SPIV) system was also utilized in the present study to quantify the generation and evolution of the streamwise vortex structures for the test cases with the BDSIC, in comparison to that of the baseline case. Fig. 7 shows the ensemble-averaged results of the SPIV measurements in several cross planes (i.e. within $Y-Z$ plane) normal to the incoming mainstream flow. As shown clearly in Fig. 7 (a), a pair of CRVs was found to be generated behind the coolant injection hole, which is found to be the dominant vortex structures for the baseline case. Obviously, as induced by the CRVs, the near-wall coolant stream would roll up and eject away from the test plate, causing the formation of strong upwash flow. As for the BDSIC case, while a similar pair of counter-rotating vortices was found to form behind the BDS shell, the rotating direction of the induced vortices was found to be opposite to those of the CRVs of the baseline case. Thus, the vortex structures are referred to as Anti-CRVs. Similar vortex pairs were also reported by Zheng et al. [30] and Omidyeganeh et al. [31]. The Anti-CRVs generated for the BDSIC case were found to induce continuous entrainment of coolant stream back to the protected surface, leading to the formation of downwash flows behind the BDS shell. As a result, the coolant jet stream exhausted from the BDS shell would spread widely on the surface of interest, and achieve largely improved film cooling effectiveness as revealed in Fig. 5. It is worth noting that, with the assistance of the BDS shell, the strong CRV pairs formed behind the conventional circular hole were found to almost disappear for the BDSIC case. This phenomenon is believed to be closely related to the unique flow features induced by BDS shell, where the coolant jet stream would discharge tangentially from the BDS shell and remain attached firmly on the surface of plate.

Figure 7(b) shows the evolution of the ensemble-averaged streamwise vorticity contours for the two compared cases at three typical downstream locations (i.e., at the downstream locations of $X / D=1.0,5.0$, 10.0 ). Apparently, the vorticity field for the baseline case was characterized by a pair of strong CRVs formed behind the circular injection hole, which was found to be the dominant flow features for the JICF pertinent to film cooling as reported in the previous studies [1,32]. Due to the viscous effects, the streamwise vortex structures were found to dissipate gradually as they traveled downstream. For the test case with BDSIC, a pair of anti-CRVs were found to be generated and congregate at each side of the BDS shell, rather than staying together within the coolant jet stream as those observed for the baseline case. It can also be seen that, while a weak pair of CRV structures was found at the downstream location of $X /$ $D=5.0$ for the BDSIC case, they were found to disappear almost completely at the downstream location of $X / D=10.0$ due to the cancellation of the vortex induction caused by the anti-CRV pair.
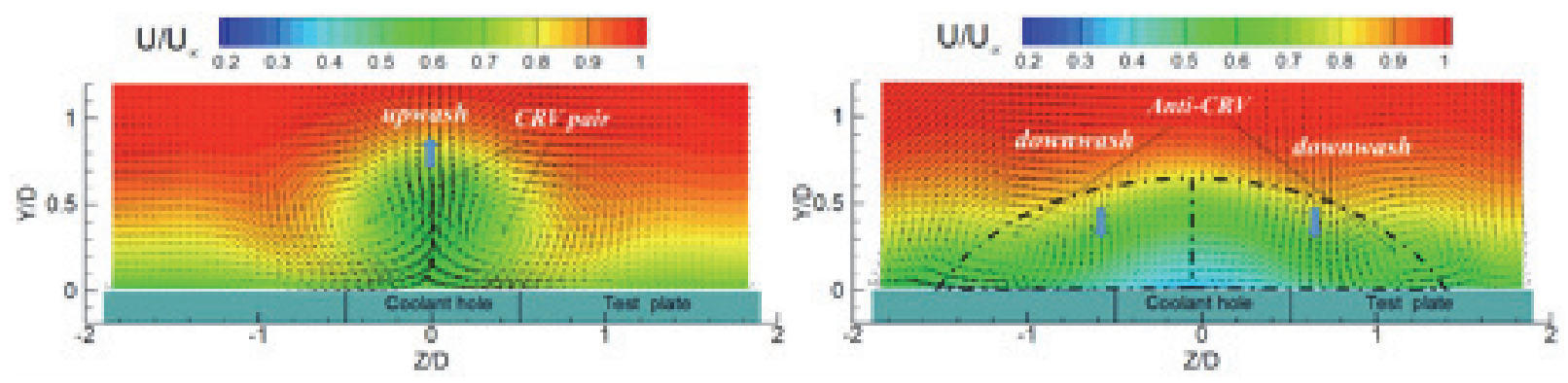
(a). ensemble-averaged velocity fields at the cross section of $X / D=5.0$.
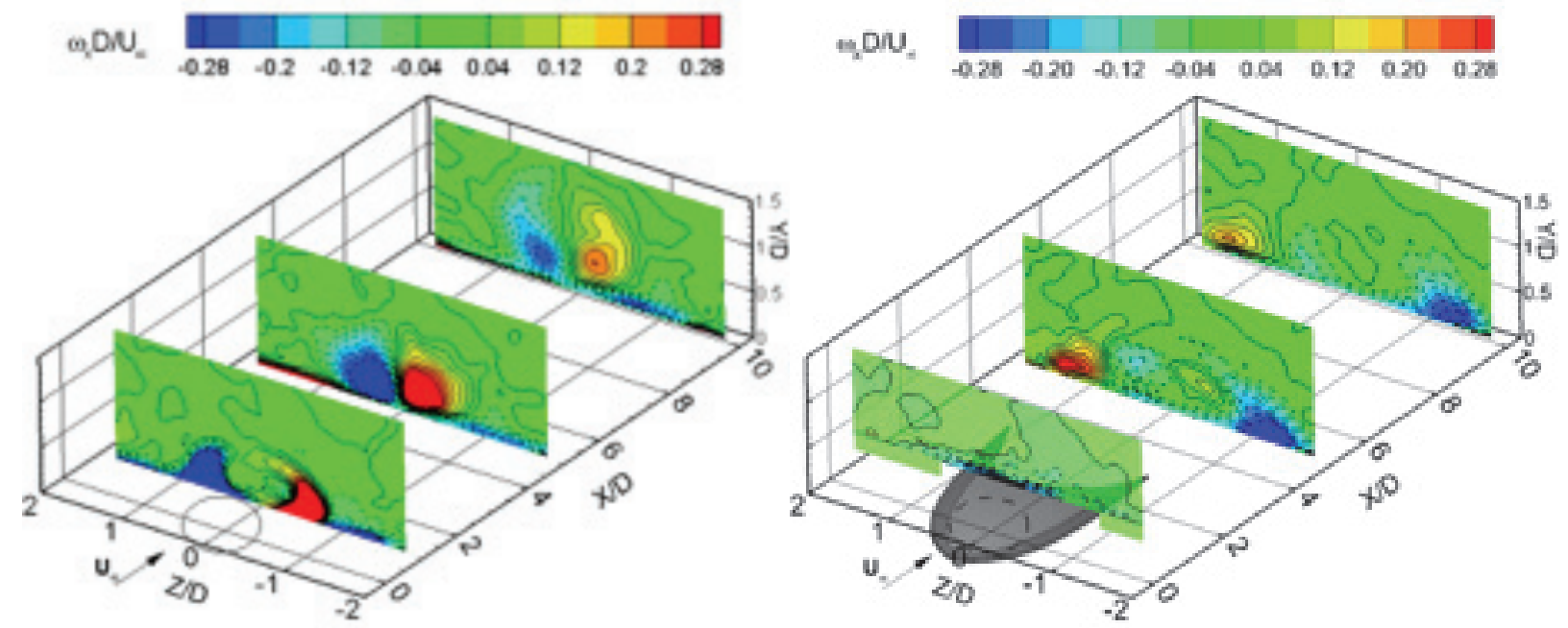

(b). evolution of streamwise vorticity distribution at three typical downstream cross sections
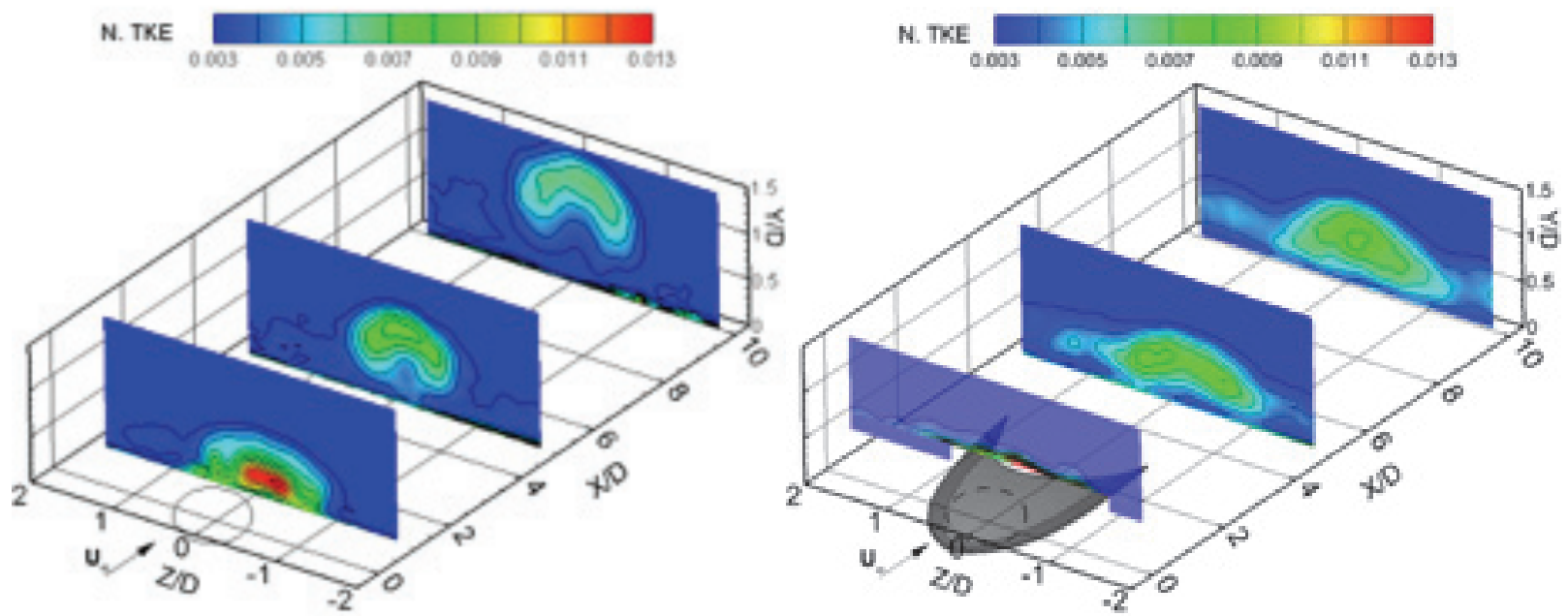

(c). evolution of the normalized TKE ditributions at three typical downstream cross sections

Figure 7. Stereoscopic PIV measurement results for baseline (left) and BDSIC cases at $M=0.9$ and $D R=1.5$ :

Figure 7(c) shows the evolution of the normalized Turbulent Kinetic Energy (TKE) distributions for the baseline case with a conventional circular hole and the BDSIC cases at various downstream locations. As revealed clearly in Fig. 7(c), in the near-hole region (i.e., $X / D=1.0$ ), the magnitude of normalized $T K E$ for the baseline case was found to be significantly higher than that of the BDSIC case with the elongated BDS shell, which is believed to be caused by the intensive interaction between the coolant and mainstream flows. Then, the N.TKE was found to decrease to a similar level with that of the BDSIC case at the downstream location of $X / D=5.0$. However, as the coolant stream traveled further downstream (i.e., $X / D=10.0$ ), the jet flow for the baseline case was found to take off from the surface and mix intensively with the mainstream flow, leading to a slightly lower N.TKE level, in comparison to that of the BDSIC case. It is worth noting that, the evolution of N.TKE shown in Fig. 7 (c) also confirmed the detachment of the coolant jet stream from the surface of interest for the baseline case, while the coolant flow was still found to concentrate in the region near the surface of the test plate for the BDSIC case. Such a conclusion was found to be consistent with that revealed from the 2-D PIV measurement results as shown in Fig. 6(b). 
In the present study, a sketch of the flow features behind the BDSIC was conjectured based on the 2D PIV and SPIV measurement results given in Fig. 6 and Fig. 7, which is shown in Fig. 8. This figure vividly illustrates the unique flow features associated with novel BDSIC design for improved film cooling effectiveness. It can be used to uncover the underlying physics pertinent to the better film cooling performance for the BDSIC designs. As shown clearly in the diagram, the flow features behind the BDSIC are relatively simple in comparison to the BDS ramp film cooling concept suggested in our previous study[29], featured by a tangential jet flow and a pair of large-scale streamwise vortices behind the BDS shell. Due to the unique flow features induced by the BDSIC, the jet flow exhausted from the circular coolant injection hole would impinge onto the inner surface of BDS shell at first, and then discharge tangentially into the mainstream flow. This process could suppress the take-off of the coolant jet flow and make it to stay firmly on the surface of interest, therefore, significantly improving the film cooling performance. The large-scale streamwise vortices generated behind the BDS shell were found to be anti-CRVs, which would induce a downwash flow to ensure the coolant stream spreading widely along the lateral direction over the surface of test plate. Consequently, the values of the film cooling effectiveness behind the BDSIC were found to become much higher than those of the baseline case. In summary, the augmentation of the film cooling effectiveness for the test case with BDSIC as observed in Fig. 5 is believed to be due to the combined effects of the tangential coolant flow injection over the surface of the test plate and the downwash flow induced by the anti-CRVs generated behind the BDS shell.

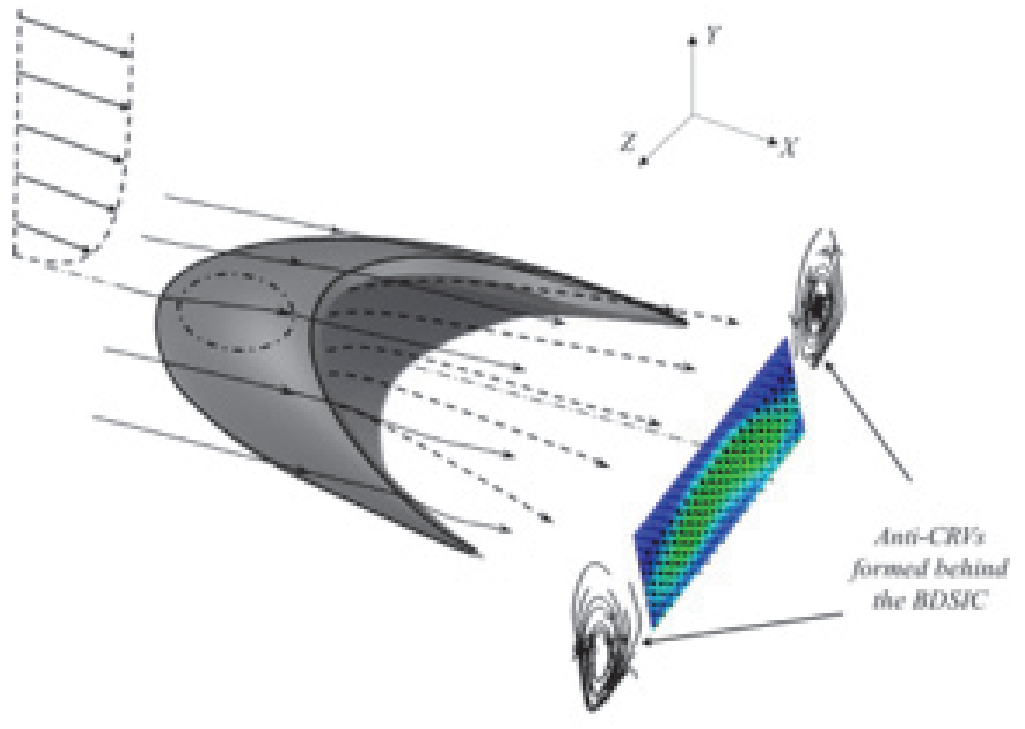

Figure 8. Schematic of the vortex structures behind a BDSIC, where the solid lines represent the streamlines of mainstream flow, the dash lines represent the streamlines of coolant stream.

\subsection{The effects of blowing ratio on the film cooling effectiveness behind the BDSIC.}

In order to examine the effects of blowing ratio on the effectiveness of film cooling performance of the BDSIC design, a series of experiments were performed at the blowing ratio of $M=0.6,0.9,1.2$, and 1.5, respectively, and the PSP measurements are shown in Fig. 9. It is apparent that, the measured film cooling effectiveness behind the BDSIC, including both centerline and laterally-averaged profiles, were found to increase monotonously as the blowing ratio increase. With the existence of the BDS shell, the coolant jet stream, unlike that of the baseline case to separate from the surface of the test plate, was found to stay firmly over the surface of the test plate disregarding its momentum augmentation. It should also be noted that, the improvement in the film cooling effectiveness for the BDSIC design was found to become smaller and smaller as the blowing ratio increases. This is believed to be due to the combined effects of the increased blowing ratio and more intensive turbulent mixing between the coolant and mainstream flows at higher blowing ratios. While an increase in the blowing ratio value would demand more coolant gas for film cooling, i.e., to provide a better protection to the surface of the test plate, this 
beneficial effect would be counteracted by the more intensive mixing between the coolant and mainstream flows, leading to an increased entrainment of the "hot" gas from outside to the region near the surface of the test plate. As a result, the improvements in the film cooling effectiveness behind the BDSIC was found to become smaller and smaller for as the blowing ratio increases.
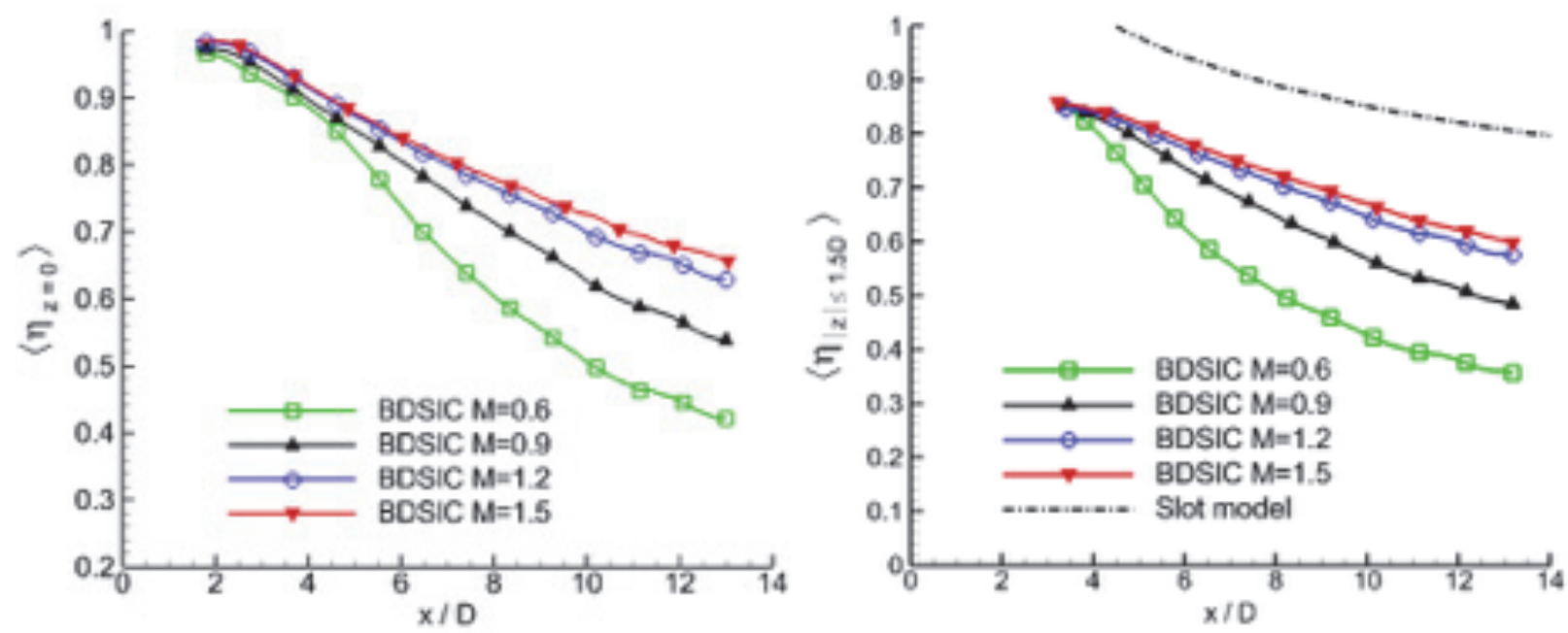

Figure 9. The measured cooling effectiveness profiles at different blowing ratios.

In order to make a quantitative comparison of the film cooling effectiveness between the proposed BDSIC design and the idealized slot film cooling, a theoretical model as suggested by Ballal and Lefebvre $[33,34]$ was used to estimate the film cooling performance of the tangential coolant injection from an idealized slot, which can be estimated by using following equation,

$$
\eta=1.28\left(\frac{\mu_{c}}{\mu_{\infty}}\right)^{0.15}\left(\frac{X}{s}\right)^{-0.2}\left(\frac{t}{s}\right)^{-0.2} \text { for } 1.3 \leq M \leq 4.0
$$

where $s$ is the height of slot (assuming the slot has a same exit area with the BDS shell, i.e., ${ }^{\pi D / 8}$ ), $\mu_{c}$ and $\mu_{\infty}$ are the dynamic viscosity of coolant and mainstream flows, respectively, and $t$ is the lip thickness (i.e., $0.1 D$ in the present study).

The film cooling effectiveness values of the idealized slot film cooling are also plotted in Fig. 9 as the dashed lines for quantitative comparison. It can be seen clearly that, as expected, the film cooling effectiveness values for the idealized slot film cooling were found to be higher than those of the BDSIC configuration. It implies that, for a fixed amount of the coolant gas, the best cooling strategy would be to have coolant flow spreading uniformly over the surface of test plate, i.e., being an idealize tangential slot flow for film cooling [35]. While the coolant stream exhausted from the BDSIC was found to tangentially flow out of the BDS shell, the distribution of the coolant stream over the surface of the test plate was found to be not as uniform as the idealized slot jet flow. Consequently, the film cooling effectiveness values behind the BDSIC were found to be smaller than those of the idealized slot film cooling.

\subsection{The effects of the neighboring BDSICs on the film cooling effectiveness.}

The film cooling for a gas turbine is usually achieved by injecting coolant flow from arrays of cooling injection holes, rather than from only a single hole. In the present study, the film cooling effectiveness 
over the surface of interest was also measured for the test case with an array of BDSICs (i.e., with a pitch of $p / D=3$ ), in comparison to that with a single BDSIC (i.e., with the pitch of $p / D=\infty$ ), in order to examine the effects of the pitch between neighboring BDSICs on the film cooling performance. The measurement results are shown in Fig. 10. It can be seen clearly that, in comparison to the test case with an array of BDSICs, while the film cooling effectiveness along the centerline of the cooling injecting hole was found to be slightly higher for the single BDSIC case (i.e., $p / D=\infty$ ), the laterally-averaged cooling effectiveness for the single BDSIC case was found to be marginally lower than that of the BDSIC array case. Due to the interactions of the adjacent anti-CRVs (i.e., as shown in Fig. 7) induced by the BDS shells in the regions between the neighboring BDSICs, the coolant jet streams for the BDSIC array case were found to coalesce with each, leading to an increased laterally-averaged cooling effectiveness but lower centerline cooling effectiveness values. This phenomenon can be seen more quantitatively from the measured lateral cooling effectiveness profiles, as shown in Fig. 10 (c) and Fig. 10(d), where the profiles were obtained by extracting the cooling effectiveness distributions at the downstream locations of $X / D=6$ and $X / D=10$, respectively.

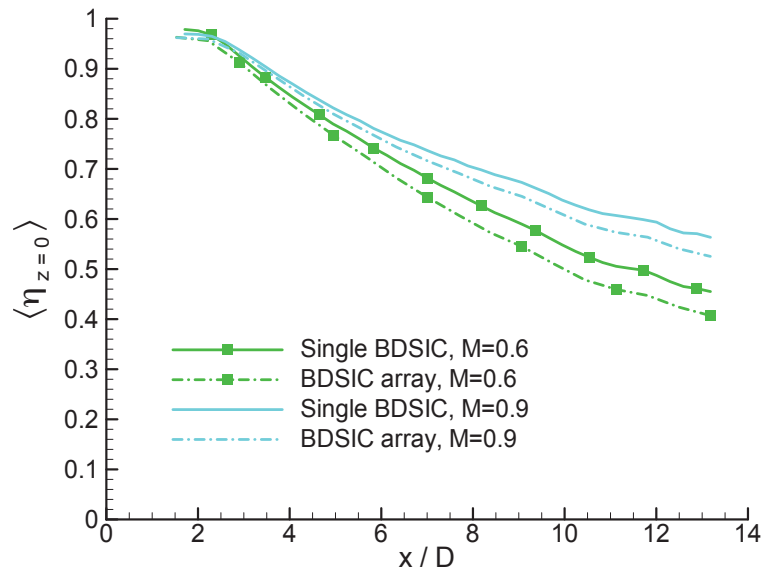

(a). Centerline cooling effectiveness profiles

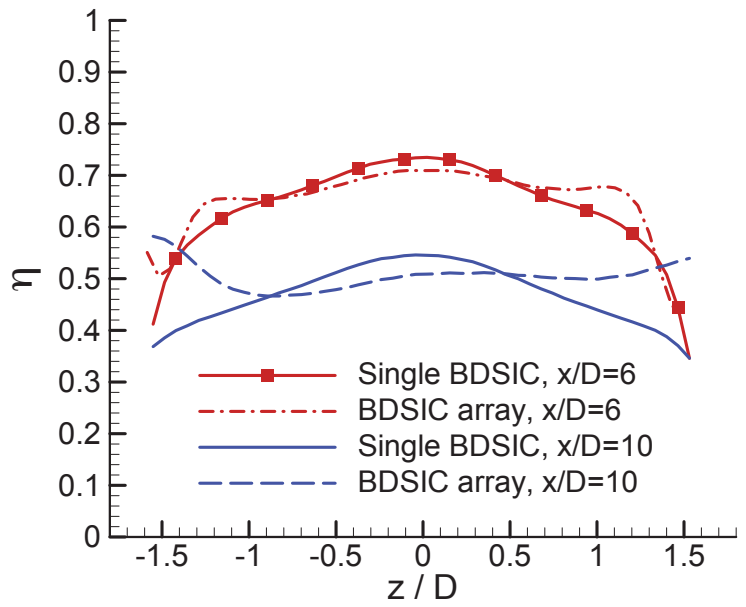

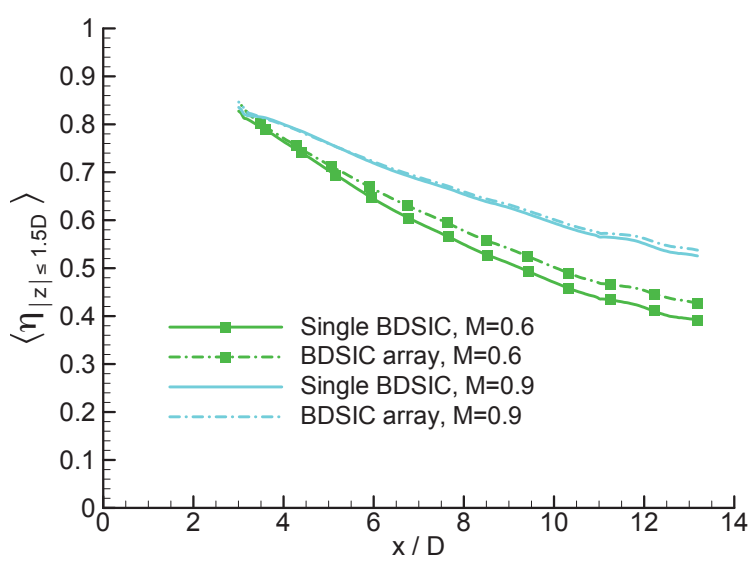

(b) laterally-averaged cooling effectiveness profiles

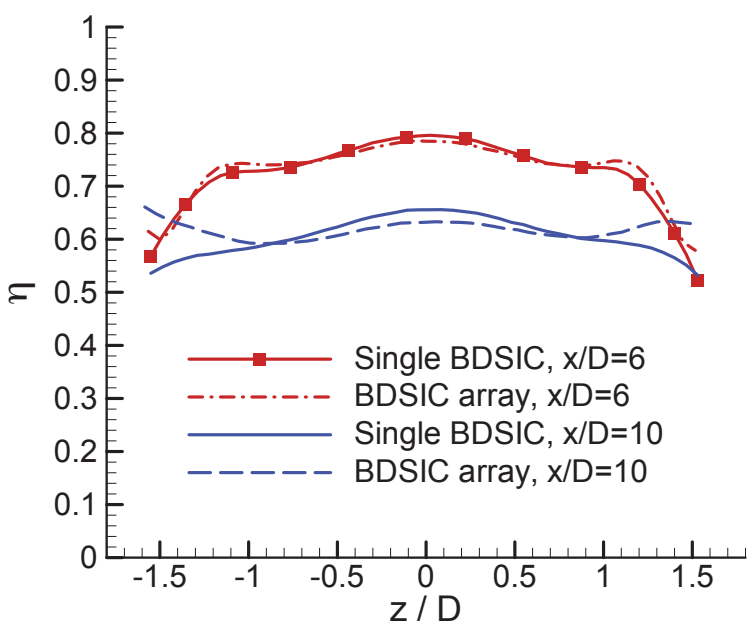

(c). Lateral cooling effectiveness profiles at $M=0.6$. (d). Lateral cooling effectiveness profiles at $M=0.9$

Figure 10. Effects of the pitch between the adjunct BDSICs on the film cooling effectiveness

\subsection{Aerodynamic loss of the film cooling with the BDSIC design}


In the present study, the aerodynamic loss of the film cooling for the BDSIC case was also estimated in order to gain further insight into the nature of the aerodynamic loss associated with the film cooling of the BDISC design. It is well known that a number of methods have been suggested to estimate the aerodynamic loss pertinent to film cooling phenomena, including the methods based on thermodynamic loss, entropy loss, total pressure loss, and so on. Further information about the aerodynamic loss estimation of different film cooling designs can be found in the review paper of Ligrani [36]. In the present study, the aerodynamic loss of the BDSIC design was estimated by using the kinetic energy loss method as suggested by Sargison[37], which is based on the estimation of the aerodynamic penalty of an film cooling design by taking the energy component from the coolant jet flow into the account.

Following the work of Sargison[37], the aerodynamic loss coefficient of a film cooling design can be estimated based on the following equation:

$$
\text { Loss }=1-\frac{0.5 \rho u_{2}^{3} A}{\frac{\sqrt{2}}{\rho_{m}}\left(P_{o m}-P_{2}\right)+\frac{m_{c}}{\rho_{c}}\left(P_{o c}-P_{2}\right)},
$$

where $A$ is the exit area of a pre-selected control volume (i.e., $15 D^{2}$ ); ${ }^{\rho}$ is the density of mixed flow at the exit of the control volume; $\rho_{m}$ is the density of mainstream flow; $\rho_{c}$ is the density of coolant flow; $u_{2}$ is the velocity at the exit of the control volume (i.e., bulk velocity was used here); $P_{2}$ is the wall pressure at the exit of the control volume; is the total pressure of mainstream; and $P_{o c}$ is the total pressure of coolant stream.

In the present study, the kinetic energy balance in a control volume within a confined cuboid of $-2 D \leq$ $X \leq 10 D, 0 \leq Y \leq 2.5 D$, and $-3 D \leq Z \leq 3 D$ was selected. The aerodynamic loss coefficients were obtained by comparing the energy difference between the 'in' and 'out' of the control volume. During the experiments, the total pressure of the coolant stream $\left(P_{o c}\right)$ was monitored by using four pressure taps which were installed around the plenum. In the meaning time, the wall pressure at the exit of the control volume was also measured by utilizing a row of pressure taps. It should be noted that the coolant stream used in the aerodynamic loss estimation is air for the present study, i.e., with a density ratio of $D R=1.0$.

Figure 11 shows the measured aerodynamic loss coefficients at various blowing ratios for the BDSIC film cooling design, in comparison with those of the baseline case (i.e., conventional circular hole). As expected, the BDSIC design was found to exhibit slightly higher (i.e., about 4\%) aerodynamic loss at relative low blowing ratios of $M \leq 0.6$, which is due to the increased friction loss associated with its complex shape. However, as the blowing ratio increases to the higher values of $M=0.9,1.2$ and 1.5, the differences in aerodynamic loss between the two compared cases were found to become smaller and smaller, and the baseline case was found eventually to have a greater aerodynamic loss in comparison to the BDSIC design at the high blowing ratio of $M=1.3$. As described above, since the coolant jet stream for the baseline case was found to take off from the surface of the test plate at relatively high blowing ratios (i.e., as shown in Fig. 6), the aerodynamic loss of the film cooling would increase rapidly due to the penetration of the cooling stream into the mainstream flow. However, the cooling stream was still found to remain staying in the region near the surface of the test plate for the BDSIC case even at relatively high blowing ratios. As a result, the aerodynamic loss for the BDSIC case was found to become even smaller than that of the baseline case at the relatively high blowing ratios. 


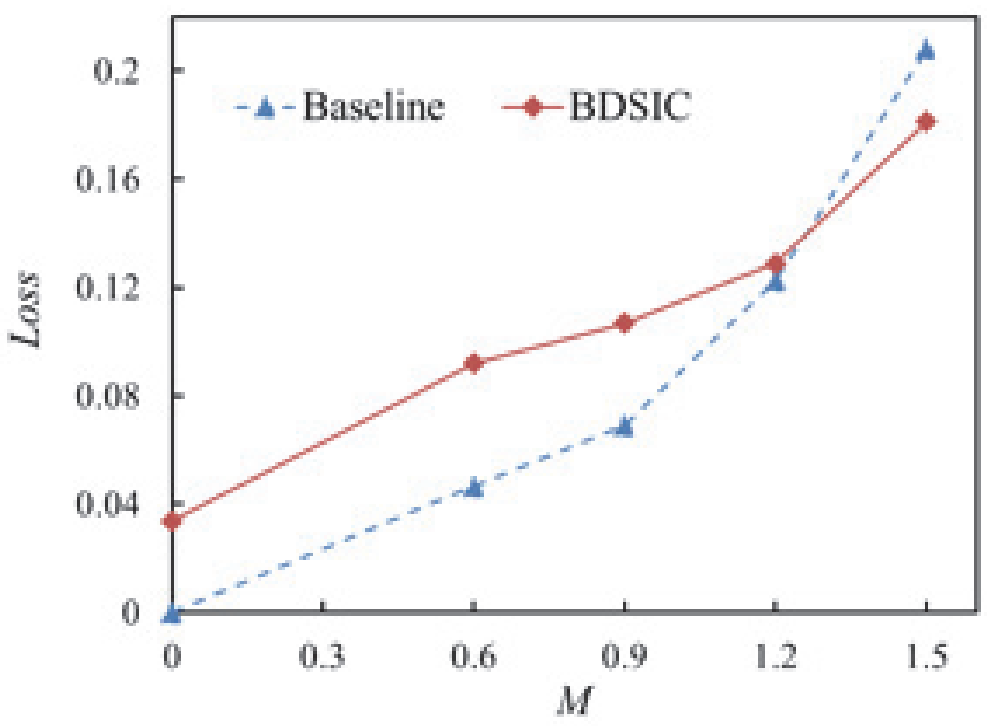

Figure 11. Comparison of the aerodynamic loss of the BDSIC design with that of the baseline case at various blowing ratios.

\section{Conclusion}

An experimental study was performed to explore a novel film cooling design with a Barchan-DuneShaped Injection Compounds (BDSIC) concept to enhance the performance of film cooling over a test plate. Inspired by the unique Barchan dune shape commonly seen in deserts, the BDSIC concept was proposed to enable the coolant stream discharged tangentially from hollowed Barchan-Dune-Shaped (BDS) shells and stay firmly on the surface of the test plate. The experimental study was conducted in a low-speed, open-circuit wind tunnel at Iowa State University. While a Pressure Sensitive Paint (PSP) technique was used to map the adiabatic film cooling effectiveness on the surface of interest, a highresolution Stereoscopic Particle Imaeg Velocimetry (PIV) system was used to conduct detailed flow field measurements to quantify the dynamic mixing process between the coolant jet and mainstream flows over the test plate. The effects of dune shape (shorted, standard, and elongated BDS shells), blowing ratio ( $M$ $=0.6,0.9,1.2,1.5$ ), and the pitch (i.e., the spanwise distance) between the neighboring DBSICs on the effectiveness of film cooling over the test plate was evaluated in great detail based on quantitative PSP and PIV measurements. The aerodynamic loss of the film cooling design with the BDSIC concept was also evaluated, in comparison to that of the conventional film cooling design with circular injection holes (i.e., the baseline case).

It was found that, with the same amount of the coolant stream exhausted from the same circular hole, the film cooling effectiveness over the test plate was found to be enhanced significantly due to the existence of the BDS shells, especially for the case with the elongated BDS shell. More specifically, in comparison with that of the baseline case, the laterally-averaged film cooling effectiveness for the BDISC case with the elongated BDS shell was found to increase by $30 \% \sim 500 \%$, as the blowing ratio was increase from $M=0.6$ to $M=1.2$. Based on the detailed PIV measurements, three distinct mechanisms were suggested to be responsible for the enhanced film cooling performance pertinent to the unique BDSIC design. Firstly, due to the unique hollowed BDS shell design, the coolant stream was discharged tangentially into the mainstream flow to stay attached to the surface of the test plate, leading to a good film cooling protection of the coolant flow to the surface of the test plate. Secondly, the dominant counter-rotating-vortex (CRV) structures usually seen in a jet-in-cross-flow (JICF) associated with conventional film cooling design with coolant injection from circular holes were found to be weakened significantly behind the BDSIC, indicating that the BDSIC design would suppress the formation of the CRV structure in the JICF and prevent the separation of the coolant stream from the surface of the test 
plate. Finally, generation of additional downwash flows (i.e., as induced by the newly formed anti-CRV structures behind the BDSIC) would entrain the coolant fluid from outside region back to near wall region over the test plate, resulting in enhanced film cooling protection over the surface of interest.

It was also found that, the film cooling effectiveness behind the BDSIC would increase monotonously as the blowing ratio increases. This is because the fact that, with the BDSIC design, the coolant jet stream would always stay attached on the surface of the test plate, in despite of the momentum augmentation of the coolant stream at higher blowing ratios. However, the improvement margin for the BDSIC design in further enhanced film cooling perfromance over the test plate was found to become smaller and smaller as the blowing ratio increases, which is believed to be closely related to the more intensive turbulent mixing between coolant and mainstream flows for the test cases with higher blow ratios. The pitch of the BDSICs (i.e., the spanwise distance between the neighboring DBSICs) was also found to have noticeable effects on the film cooling effectiveness over the test plate. In comparison to the test case with an array of BDSICs on the test plate (i.e., with the effects of the neighboring DBSICs), while the centerline cooling effectiveness values for the test case with only a single BDSIC (i.e., without the effects of the neighboring DBSICs) were found to be slightly higher, the laterally-averaged cooling effectiveness values were found to become marginally lower.

Due to a greater friction loss associated with its complex geometry, the BDSIC design was found to have a higher aerodynamic loss than the conventional film cooling design (i.e., with circular injection hole only) at relative low blowing ratios, as expected. However, since the BDSIC design would induced more complex vortex structures downstream to keep the coolant stream to flow more firmly over the surface of test plate (i.e., less aerodynamic loss associated with the penetration of the coolant stream into the mainstream flow), the aerodynamic loss of the BDSIC design was found to become even smaller than that of the conventional film cooling design at relatively high blowing ratios.

While the findings of the present study demonstrated clearly that the proposed BDSIC concept is very promising to enhance film cooling effectiveness over the surface of interest, especially for its superior film cooling augmentation peformance at relatively high blowing ratios with even less aerodynamic loss than that of the conventional film cooling design, more comprehesive investigations are still needed to elucidate the underlying physics and to exmine the relevant parameters, such as the discharge coefficients, heat transfer coefficients, the effects of the compressibility of the gaseous flows, the geometry optimization of the BDSIC design, in order to explore/optimize the design paradigms for better film cooling protection of the hot components in gas turbines from harsh enviroments.

\section{Acknowledgment}

The technical assistances of Dr. Blake Johnson, Mr. Bill Richard, and Mr. Brandon Weigel of Iowa State University are greatly appreciated. Support from the National Science Foundation with Grant numbers of CBET- 1438099 is gratefully acknowledged.

\section{Reference}

[1] T.F. Fric, A. Roshko, Vortical structure in the wake of a transverse jet, J. Fluid Mech. 279 (1994) 1-47. doi:10.1017/S0022112094003800.

[2] J.D. Heidmann, S. Ekkad, A Novel Antivortex Turbine Film-Cooling Hole Concept, J. Turbomach. 130 (2008) 031020. doi:10.1115/1.2777194.

[3] A. Dhungel, Y. Lu, W. Phillips, S. V. Ekkad, J. Heidmann, Film Cooling From a Row of Holes Supplemented With Antivortex Holes, J. Turbomach. 131 (2009) 021007. doi:10.1115/1.2950059.

[4] K.B.M.Q. Zaman, J.K. Foss, The Effect of Vortex Generators on a Jet in a Cross-Flow, Phys. Fluids. 9 (1997) 106-114. http://ntrs.nasa.gov/search.jsp?R=19980202675. 
[5] K.M. Zaman, D. Rigby, J. Heidmann, Inclined Jet in Crossflow Interacting With a Vortex Generator, J. Propuls. Power. 26 (2010) 947-954. http://arc.aiaa.org/doi/pdf/10.2514/1.49742.

[6] A.F. Shinn, S. Pratap Vanka, Large eddy simulations of film-cooling flows with a micro-ramp vortex generator, J. Turbomach. 135 (2013) 011004. http:// tribology.asmedigitalcollection.asme.org/article.aspx?articleid=1673700.

[7] S. Na, T. Shih, Increasing adiabatic film-cooling effectiveness by using an upstream ramp, J. Heat Transfer. 129 (2007) 464-471. http://pressurevesseltech.asmedigitalcollection.asme.org/ article.aspx?articleid $=1448679$.

[8] W. Zhou, H. Hu, Improvements of film cooling effectiveness by using Barchan dune shaped ramps, Int. J. Heat Mass Transf. 103 (2016) 442-456. doi:10.1016/j.ijheatmasstransfer. 2016.07.066.

[9] B. Andreotti, P. Claudin, S. Douady, Selection of dune shapes and velocities Part 1: Dynamics of sand, wind and barchans, Eur. Phys. J. B - Condens. Matter. 28 (2002) 321-339. doi:10.1140/epjb/ e2002-00236-4.

[10] E. Parteli, O. Durán, H. Herrmann, Minimal size of a barchan dune, Phys. Rev. E. 75 (2007) 011301. doi:10.1103/PhysRevE.75.011301.

[11] J.A. Palmer, R. Mejia-Alvarez, J.L. Best, K.T. Christensen, Particle-image velocimetry measurements of flow over interacting barchan dunes, Exp. Fluids. 52 (2011) 809-829. doi: 10.1007/s00348-011-1104-4.

[12] K.T. Christensen, F. Kazemifar, G. Blois, J.M. Barros, Z. Tang, A. Hamed, T. Kim, J.L. Best, Quantitative Studies of Environmental Flows at the Micro- and Macro-Scales, in: 16th Int. Symp. Flow Vis. Okinawa, Japan, 2014.

[13] B. Horvat, Barchan dunes Seminar 2, (n.d.). http://www-f1.ijs.si/ rudi/sola/Sem4.pdf.

[14] P. Hersen, K. Andersen, H. Elbelrhiti, B. Andreotti, P. Claudin, S. Douady, Corridors of barchan dunes: Stability and size selection, Phys. Rev. E. 69 (2004) 011304. doi:10.1103/PhysRevE. 69.011304.

[15] E.R.G. Eckert, Similarity analysis of model experiments for film cooling in gas turbines, WärmeUnd Stoffübertragung. 27 (1992) 217-223. doi:10.1007/BF01589919.

[16] J.N. Shadid, E.R.G. Eckert, The Mass Transfer Analogy to Heat Transfer in Fluids With Temperature-Dependent Properties, J. Turbomach. 113 (1991) 27. doi:10.1115/1.2927734.

[17] L.M. Wright, Z. Gao, T.A. Varvel, J.-C. Han, Assessment of Steady State PSP, TSP, and IR Measurement Techniques for Flat Plate Film Cooling, in: Heat Transf. Vol. 3, ASME, 2005: pp. 37-46. doi:10.1115/HT2005-72363.

[18] J.H. Bell, E.T. Schairer, L.A. Hand, R.D. Mehta, SURFACE PRESSURE MEASUREMENTS USING LUMINESCENT COATINGS 1, Annu. Rev. Fluid Mech. 33 (2001) 155-206. doi: 10.1146/annurev.fluid.33.1.155.

[19] B. Johnson, W. Tian, K. Zhang, H. Hu, An experimental study of density ratio effects on the film cooling injection from discrete holes by using PIV and PSP techniques, Int. J. Heat Mass Transf. 76 (2014) 337-349. doi:10.1016/j.ijheatmasstransfer.2014.04.028.

[20] D. Charbonnier, P. Ott, M. Jonsson, F. Cottier, T. Köbke, Experimental and Numerical Study of the Thermal Performance of a Film Cooled Turbine Platform, in: Asme Turbo Expo 2009, Vol 3, Pts A B, ASME, 2009. doi:10.1115/GT2009-60306.

[21] Z. Yang, H. Hu, Study of Trailing-Edge Cooling Using Pressure Sensitive Paint Technique, J. Propuls. Power. 27 (2011) 700-709. doi:10.2514/1.B34070.

[22] B. Johnson, H. Hu, Measurement Uncertainty Analysis in Determining Adiabatic Film Cooling Effectiveness by Using Pressure Sensitive Paint Technique, J. Turbomach. 138 (2016) 121004. 
doi:10.1115/1.4033506.

[23] M. Sajben, Uncertainty estimates for pressure sensitive paint measurements, AIAA J. 31 (1993) 2105-2110. doi:10.2514/3.11897.

[24] S.J. Kline, F.A. Mcclintock, Describing uncertainties in single sample experiments, J. Mech. Eng. 75 (1953).

[25] H. Hu, T. Saga, T. Kobayashi, N. Taniguchi, A study on a lobed jet mixing flow by using stereoscopic particle image velocimetry technique, Phys. Fluids. 13 (2001) 3425. doi: 10.1063/1.1409537.

[26] L. Yang, P. Ligrani, J. Ren, H. Jiang, Unsteady Structure and Development of a Row of Impingement Jets, Including Kelvin-Helmholtz Vortex Development, ASME J. Fluids Eng. 137 (2015) 051201.

[27] W. Zhou, Y. Rao, H. Hu, An Experimental Investigation on the Characteristics of Turbulent Boundary Layer Flows Over a Dimpled Surface, J. Fluids Eng. 138 (2015) 021204. doi: 10.1115/1.4031260.

[28] S.D. Peterson, M.W. Plesniak, Evolution of jets emanating from short holes into crossflow, J. Fluid Mech. 503 (2004) 57-91. doi:10.1017/S0022112003007407.

[29] W. Zhou, H. Hu, An Experimental Study on Film Cooling Performance Behind Barchan DuneShaped Ramps Using PSP and PIV Techniques, in: Vol. 5B Heat Transf., ASME, 2015: p. V05BT12A043. doi:10.1115/GT2015-43781.

[30] Y. Zheng, A. Rinoshika, Multi-scale vortical structure analysis on large eddy simulation of dune wake flow, J. Vis. 18 (2015) 95-109. doi:10.1007/s12650-014-0227-0.

[31] M. Omidyeganeh, U. Piomelli, K.T. Christensen, J.L. Best, Large-eddy simulation of flow over barchan dunes, in: Mar. River Dune Dyn. - MARID IV, Bruges, 2013: pp. 191-198.

[32] D.R. Getsinger, L. Gevorkyan, O.I. Smith, A.R. Karagozian, Structural and stability characteristics of jets in crossflow, J. Fluid Mech. 760 (2014) 342-367. doi:10.1017/jfm.2014.605.

[33] D. Ballal, A. Lefebvre, A proposed method for calculating film-cooled wall temperatures in gas turbine combustion chambers, 1973.

[34] A.H. Lefebvre, D.R. Ballal, Gas turbine combustion; alternative fuels and emissions, 3rd ed., CRC Press, 2010. http://cds.cern.ch/record/1611397.

[35] R. Goldstein, Film cooling, in: Adv. Heat Transf. 7.1, 1971: pp. 321-379.

[36] P. Ligrani, Aerodynamic Losses in Turbines with and without Film Cooling, as Influenced by Mainstream Turbulence, Surface Roughness, Airfoil Shape, and Mach, Int. J. Rotating Mach. 2012 (2012) 28. doi:10.1155/2012/957421.

[37] J.E. Sargison, Development of a Novel Film Cooling Hole Geometry, PhD Dissertation, Dept. of Engineering Sci., University of Oxford, 2001. 